\title{
Continuous time dynamics of the Thermal Minority Game
}

\author{
Juan P. Garrahan日, Esteban Morol and David Sherringtond \\ Theoretical Physics, University of Oxford, 1 Keble Road, Oxford OX1 3NP, United Kingdom
}

(April 17, 2000)

\begin{abstract}
We study the continuous time dynamics of the Thermal Minority Game. We find that the dynamical equations of the model reduce to a set of stochastic differential equations for an interacting disordered system with non-trivial random diffusion. This is the simplest microscopic description which accounts for all the features of the system. Within this framework, we study the phase structure of the model and find that its macroscopic properties strongly depend on the initial conditions.
\end{abstract}

Many of the current challenges for statistical physics have their origins in problems in biology [1] and economics [2:3]. In particular, the application of ideas and techniques of the statistical mechanics of disordered systems can prove useful in the study of systems of adaptive and competitive agents, which are relevant, for example, to the microscopic modeling of financial markets; and, conversely, such problems can raise new issues for statistical physics. One of these systems is the minority game (MG) 44.5, a simple model based on Arthur's "El Farol" bar problem [6] which describes the behaviour of a group of competing heterogeneous agents subject to the economic law of supply and demand. Despite its simplicity, the MG is very non-trivial, and although much progress has been made in the qualitative [7] 9] and quantitative 10,11 understanding of its features, a full analytic solution of the MG is still missing.

The main hurdles in the way of an analytical study of the MG in its original formulation were its non-locality in time due to the dependence on the game history, its discrete kinematics and dynamics, and the "best-strategy" rule (see, however, [10]). The first of these obstacles was overcome in [12], where it was shown numerically that the macroscopic behaviour of the MG was unchanged if the real history was replaced by a random one. This allowed the study of a simpler stochastic Markovian problem instead of the original deterministic non-Markovian one.

In 13. a natural continuous generalization of the MG was presented. The "information" to which the agents reacted was taken as an external input to the system and it was shown that all the macroscopic features of the MG were preserved, as long as the external information was ergodic in time, the simplest choice being just noise. To handle the problem of the 'best-strategy' rule, the Thermal Minority Game (TMG) was introduced, in which a certain degree of stochasticity in the choice of the strategies by the agents was allowed, controlled by a parameter $T$, the "temperature", the limits $T=0$ and $T=\infty$ corresponding to the original deterministic MG and the case of completely random strategy choices, respectively. The TMG displayed extra non-trivial structure as a function of $T$, notably that in the region where the MG performs worse than random, the system can be made to perform better than random by allowing a certain degree of indi- vidual stochastic error.

In the present paper we carefully study the continuous time limit of the TMG, in order to obtain the simplest microscopic description which accounts for all the macroscopic features of the system, and as a further step towards an analytical solution of the model. We confirm that the external information 'integrates-out' providing simply an effective coupling between agents. We also show the crucial dependence of the macroscopic properties of the model on the initial conditions. We find that the microscopic equations of the TMG reduce to a set of disordered stochastic differential equations with a non-trivial random diffusion matrix, and study the phase structure of the model in the temperature/dimension plane.

The setup of the TMG is as follows [13]. The system consists of $N$ agents playing the game. At each time step $t$, each agent reacts to a common piece of "information" $\vec{I}(t)$, by making a "bid" $b_{i}(t)(i=1, \ldots, N)$. The information, defined as a unit-length vector in $\mathbb{R}^{D}$, is taken to be a random noise, $\delta$-correlated in time and uniformly distributed on the unit sphere 14]. The bid $b(t)$ is defined to be a real number, which can be interpreted as placing an order in a market, of size $|b(t)|$ and positive/negative meaning buy/sell. Bids are prescribed by "strategies": maps from information to bid, $\mathbb{R}^{D} \rightarrow \mathbb{R}$. For simplicity the strategy space $\Gamma$ of the model is restricted to the subspace of homogeneous linear mappings. A strategy $\vec{R}$ is defined as a vector in $\mathbb{R}^{D}$, subject to the constraint $\|\vec{R}\|=\sqrt{D}$, and the prescribed bid is just the scalar product $\vec{R} \cdot \vec{I}(t)$. Each agent has $S$ strategies, drawn randomly and independently from $\Gamma$ (with uniform distribution) remaining fixed throughout the game. In what follows we will restrict for simplicity to the case of two strategies per agent $S=2$, the generalization to $S>2$ being straightforward. At time step $t$ each agent $i$ chooses one of his/her strategies $\vec{R}_{i}^{\star}(t)$ to play with. The "total bid" (or "excess demand") is then $A(t) \equiv \sum_{j} b_{j}(t)=\sum_{j} \vec{R}_{j}^{\star}(t) \cdot \vec{I}(t)$. The agents keep track of the potential success of the strategies by assigning points to them, which are updated according to $P(\vec{R}, t+1)=P(\vec{R}, t)-A(t) b(\vec{R}) / N$, where $P(\vec{R}, t)$ represents the points of strategy $\vec{R}$ at time $t$. 
In the original formulation of the MG the agents played in a deterministic fashion using their 'best' strategies, the ones with the highest number of points. In the TMG the natural generalization to non-deterministic behaviour is allowed. At time step $t$, each agent $i$ chooses $\vec{R}_{i}^{*}(t)$ randomly from his/her $\left\{\vec{R}_{i}^{1}, \vec{R}_{i}^{2}\right\}$ with probabilities $\left\{\pi_{i}^{1}(t), \pi_{i}^{2}(t)\right\}$. The probabilities $\pi_{i}^{a}(t)$ are functions of the points parameterized by a temperature $T$, defined so as to interpolate between the MG case at $T=0$, all the way up to the totally random case $\pi_{i}^{1}=\pi_{i}^{2}=1 / 2$ at $T=\infty$. The qualitative behaviour of the system does not depend on the specific functional form of the $\pi_{i}^{a}(t)$. In 13 the probabilities were defined as $\pi_{i}^{1,2}(t) \propto \exp \left[\beta P\left(\vec{R}_{i}^{1,2}, t\right)\right]\left(\right.$ with $\pi_{i}^{1}(t)+\pi_{i}^{2}(t)=1$ and $\beta=1 / T$ ), while an alternative convenient form is given by $\pi_{i}^{1,2}(t) \propto \exp \left[ \pm \beta z_{i}(t)\right]$, where $z_{i}(t) \equiv \operatorname{sgn}\left(p_{i}(t)\right)$, with $p_{i}(t) \equiv\left[P\left(\vec{R}_{i}^{1}, t\right)-P\left(\vec{R}_{i}^{2}, t\right)\right] / 2$. The consequential difference between these two definitions will be discussed below.

The set of unconstrained degrees of freedom of the TMG is given by the difference $p_{i}(t)$ of the points of the two strategies of each agent. The choice of strategies can then be defined by $\vec{R}_{i}^{*}(t)=\vec{h}_{i}+\vec{\xi}_{i} \operatorname{sgn}\left[s_{i}(t)+\mu(t)\right]$, where $s_{i}(t) \equiv \pi_{i}^{1}(t)-\pi_{i}^{2}(t), \vec{\omega}_{i} \equiv\left(R_{i}^{1}+R_{i}^{2}\right) / 2, \vec{\xi}_{i} \equiv$ $\left(R_{i}^{1}-R_{i}^{2}\right) / 2$, and $\mu(t)$ is a stochastic random variable uniformly distributed between -1 and 1 and independently distributed in time. The equations for the point differences then read,

$$
p_{i}(t+1)=p_{i}(t)-\vec{a}(t) \cdot \vec{I}(t) \vec{\xi}_{i} \cdot \vec{I}(t),
$$

where $\vec{a}(t) \equiv \sum_{i} \vec{R}_{i}^{\star}(t) / N$. Eqs. (1), together with the random processes for $\vec{I}(t)$ and $R_{i}^{*}(t)$, define the dynamics of the TMG.

The average of the total bid $A(t)$ over time and quenched disorder is zero, so the first relevant macroscopic observable of the TMG is its normalized standard deviation $\sigma$ (or "volatility") $\sigma^{2} \equiv N^{-1} \overline{\left\langle A^{2}(t)\right\rangle}$, where the overline means disorder average, and $\langle\cdot\rangle \equiv$ $\lim _{t \rightarrow \infty} \frac{1}{t} \int_{t_{0}}^{t_{0}+t}(\cdot) d t^{\prime}$. In 13] it was found that $\sigma$ had a non-trivial structure both as a function of the reduced dimension of the strategy space $d=D / N$ and of the temperature. The second important observable is the fraction $\phi$ of "frozen" agents, defined as those for which one of the strategies always outperforms the other, $\phi \equiv N^{-1} \sum_{i} \delta\left(\left|\left\langle z_{i}(t)\right\rangle\right|-1\right)$, with the normalization $\delta(0)=1$. It was introduced in 10 as an order parameter for the MG, where it was found that $\phi(d)$ changed from zero to a finite value at $d=d_{c}$.

We now consider the continuous time limit of Eqs. (1) in such a way as to preserve all the macroscopic features of the TMG. To this end we introduce an arbitrary time step $\Delta t$. We deal first with the information $\vec{I}(t)$. Let us assume that $\vec{I}(t)$ is a differential random motion in the space of strategies, i.e., $\vec{I}(t)=\Delta \vec{W}(t)$, with zero mean and variance $\Delta t$. Replacing in Eqs. (11) we obtain $p_{i}(t+\Delta t)=p_{i}(t)-\vec{a}(t) \cdot \Delta \vec{W}(t) \overrightarrow{\xi_{i}} \cdot \Delta \vec{W}(t)$. In the limit $\Delta t \rightarrow 0$, and using the Kramers-Moyal expansion [15], we get

$$
d p_{i}(t)=-\vec{a}(t) \cdot \vec{\xi}_{i} d t / D+\mathcal{O}\left(d t^{2}\right) .
$$

Note that to $\mathcal{O}(d t)$ the noise has been eliminated in favour of an effective interaction among the agents, and the $\sigma$ becomes $\sigma^{2}=(N D)^{-1} \overline{\langle\vec{a}(t) \cdot \vec{a}(t)\rangle}$.

At $T=0$, corresponding to the MG, Eqs. (2) are completely deterministic. To first order in $d t$ we have

$$
d p_{i}(t)=-\left[h_{i}+\sum_{j} J_{i j} z_{j}(t)\right] d t,
$$

where $h_{i} \equiv \sum_{j} \vec{\omega}_{j} \cdot \vec{\xi}_{i} / N D$ and $J_{i j} \equiv \vec{\xi}_{j} \cdot \vec{\xi}_{i} / N D$, while the volatility reads

$$
\sigma^{2}=\bar{\Omega}+2 \sum_{i} \overline{h_{i}\left\langle z_{i}(t)\right\rangle}+\sum_{i j} \overline{J_{i j}\left\langle z_{i}(t) z_{j}(t)\right\rangle},
$$

and $\Omega \equiv \sum_{i j} \vec{\omega}_{i} \cdot \vec{\omega}_{j} / N D$. In order to check the continuous time approximation at $T=0$ we have simulated Eqs. (3). Results are presented in Fig. 1. We can see that this approximation reproduces all the features of the original MG. Note that in Eqs. (3) all stochasticity coming from the information has dropped out, the only effect being a small quantitative deviation in the low $d$ region.

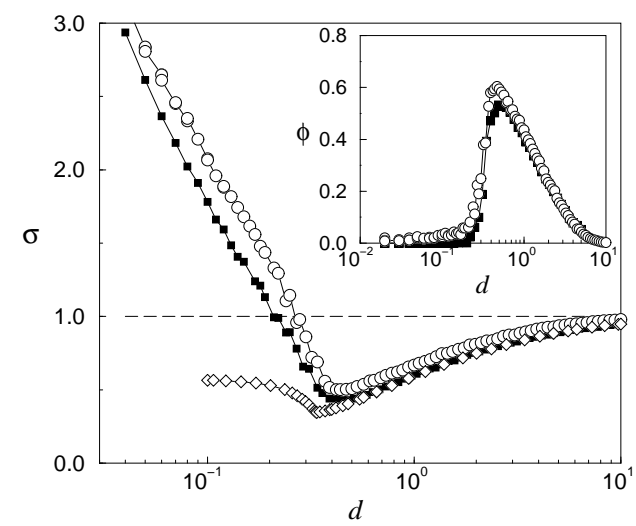

FIG. 1. Volatility $\sigma$ as a function of the reduced dimension $d=D / N$. Squares correspond to the original dynamics Eqs. (11); circles $\bigcirc$ to simulations of Eqs. (3), where an Euler algorithm has been used with time step $d t=0.05$; diamonds $\diamond$ to the approximation of [11]. In the inset we show $\phi$ as a function of $d$. Average over 100 samples; $N=100$; $t=t_{0}=10^{4}$.

Eqs. (3) can be rewritten as $d \mathbf{p} / d t=-\nabla_{\mathbf{s}} \mathcal{H}$, where $\mathbf{p} \equiv\left(p_{1}, \ldots, p_{N}\right)$, similarly for $\mathbf{s}$, and

$$
\mathcal{H}=\frac{1}{2} \Omega+\sum_{i} h_{i} s_{i}+\frac{1}{2} \sum_{i j} J_{i j} s_{i} s_{j}
$$

This is similar to what was done in [11] for the time and information averages of $z_{i}$. There the value of $\sigma$ was 
related to the average extrema of $\mathcal{H}$ by assuming that the system equilibrated. A good agreement with the numerics was found in the phase $d>d_{c}$, but this method failed to reproduce the behaviour in the $d<d_{c}$ phase (see Fig. 1). This disagreement was speculated as due to the need for terms with higher order time derivatives in the continuous time equations. This is clearly wrong, since, as we have just shown, Eqs. (3) describe correctly the dynamics of the model for all values of $d$ (see Fig. 1).

The phase $d<d_{c}$ of the MG is very sensitive to the initial conditions. In Fig. 2 we show the results of simulating both the original dynamics Eqs. (if) and the continuous time approximation Eqs. (3) starting from random initial conditions $\left|p_{i}(0)\right|=\mathcal{O}(1)$. From Fig. 2 see that the behaviour of both $\sigma$ and $\phi$ is different from that of Fig. 1 in the region $d<d_{c}$ : the system stays in the better-thanrandom phase for all values of $d$. Again the continuous time dynamics is very close to the original discrete one. This sensitivity of the results to the initial conditions is a clear indication that the system does not equilibrate for $d<d_{c}$, and raises a question on the existence and character of the "phase transition" in the MG [10].

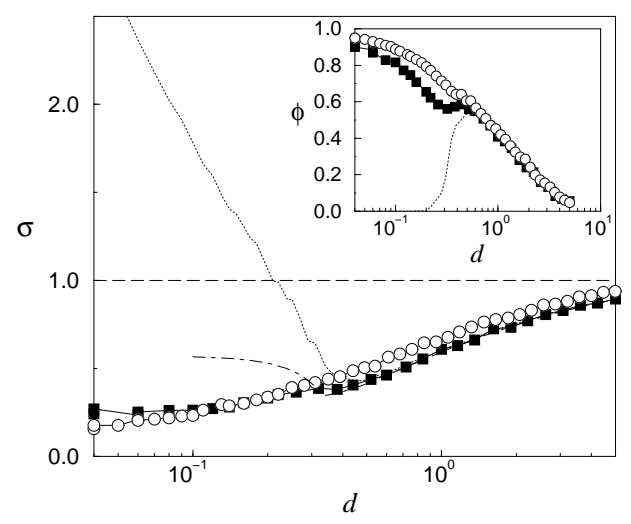

FIG. 2. Volatility as a function of $d$ for random initial conditions $\left|p_{i}(0)\right|=\mathcal{O}(1)$, for the original dynamics Eqs. (1) and the continuous time approximation Eqs. (3). Dotted lines correspond to zero initial conditions and the approximation of [11]. In the inset we show the fraction of frozen agents $\phi$. Symbols and details of the simulations are those of the previous figure.

When the temperature is different from zero the TMG Eqs. (2) still depend on the stochastic choice of strategies $R_{i}^{*}(t)$, even at leading order. At each time step, $R_{i}^{*}$ takes one of the two possible values $R_{i}^{1,2}$, defining a stochastic jump process. In order to write the corresponding Master Equation we need to know the transition probabilities. The r.h.s. of Eq. (2), which we denote $\Delta_{i}$, is a normalized sum of $N$ random numbers $\vec{\xi}_{i} \cdot R_{j}^{*}(t)$, each with mean $\vec{\xi}_{i} \cdot \vec{\omega}_{j}+\vec{\xi}_{i} \cdot \vec{\xi}_{j} s_{j}(t)$, and variance $\left(\vec{\xi}_{i} \cdot \vec{\xi}_{j}\right)^{2}\left[1-s_{j}^{2}(t)\right]$. By the central limit theorem, we know that for $N$ large $\Delta_{i}$ will tend to be normally distributed with mean $\left\langle\left\langle\Delta_{i}\right\rangle\right\rangle=\partial \mathcal{H} / \partial s_{i}$, and variance
$\left\langle\left\langle\Delta_{i}^{2}\right\rangle\right\rangle=\sum_{j} J_{i j}^{2}\left[1-s_{j}^{2}(t)\right]$, where $\langle\langle\cdot\rangle\rangle$ stands for average over realizations of the random process $\mu(t)$. Moreover, $\Delta_{i}$ and $\Delta_{j \neq i}$ are correlated, the covariance matrix given by

$$
\begin{aligned}
M_{i j}[\mathbf{p}(t)] & \equiv\left\langle\left\langle\Delta_{i} \Delta_{j}\right\rangle\right\rangle-\left\langle\left\langle\Delta_{i}\right\rangle\right\rangle\left\langle\left\langle\Delta_{j}\right\rangle\right\rangle \\
& =\sum_{k} J_{i k} J_{j k}\left[1-s_{k}^{2}(t)\right] .
\end{aligned}
$$

Collecting these results, we obtain the transition probabilities in the large $N$ limit, $W\left(\mathbf{p}^{\prime} \mid \mathbf{p}\right)=\Phi\left(\nabla_{\mathbf{s}} \mathcal{H} ; \mathbb{M}\right)$, where $\Phi$ corresponds to the normal distribution with mean $\nabla_{\mathbf{s}} \mathcal{H}$ and covariance matrix $\mathbb{M} \equiv\left\{M_{i j}\right\}$. Note that $\partial \mathcal{H} / \partial s_{i} \sim \mathcal{O}(1)$, and $M_{i j} \sim \mathcal{O}(1 / N)$, so that fluctuations are also of $\mathcal{O}(1)$ and thus are not suppressed when $N \rightarrow \infty$.

The $R_{i}^{*}(t)$ are chosen independently at each time. If we make the natural assumption that in the limit $d t \rightarrow 0$ their correlation at different times is a $\delta$-function, the Master Equation becomes a Fokker-Planck equation by means of Kramers-Moyal expansion 15]

$$
\frac{\partial \mathcal{P}}{\partial t}=-\sum_{i} \frac{\partial}{\partial p_{i}}\left(\frac{\partial \mathcal{H}}{\partial s_{i}} \mathcal{P}\right)+\frac{1}{2} \sum_{i j} \frac{\partial^{2}}{\partial p_{i} \partial p_{j}}\left(M_{i j} \mathcal{P}\right) .
$$

We therefore conclude that the dynamics of the TMG is effectively described by a set of stochastic differential equations for the point differences

$$
d \mathbf{p}=-\nabla_{\mathbf{s}} \mathcal{H} d t+\mathbb{M} \cdot d \mathbf{W},
$$

where $\mathbf{W}(t)$ is an $N$-dimensional Wiener process, and the volatility is given by $\sigma^{2}=2 \overline{\langle\mathcal{H}\rangle}+\sum_{i} \overline{J_{i i}}-\sum_{i} \overline{J_{i i}\left\langle s_{i}^{2}\right\rangle}$.

We have checked by means of extensive numerical simulations that Eqs. (8) give the same results as Eqs. (2), up to statistical errors. Figs. 3 and 1 present the results from the continuous time dynamics Eqs. (8). For these simulations we have chosen for the strategy-use probabilities the form $\pi_{i}^{1,2}(t) \propto \exp \pm \beta z_{i}(t)$ which makes the numerics simpler. Similar results can be obtained with the form $\pi_{i}^{1,2}(t) \propto \exp \beta P\left(\vec{R}_{i}^{1,2}, t\right)$, but a small- $p$ cutoff of $\mathcal{O}(d t)$ is required to avoid the system getting trapped in the $p=0$ region. In Fig. 3 we plot the volatility as a function of the temperature, showing that the behaviour is the same as the one found in 13]: for $d<d_{c}$, as $T$ is increased $\sigma$ first drops to a minimum, and then increases towards the random case $\sigma=1$; for $d>d_{c}$, the optimum value is the MG one, and $\sigma$ simply grows monotonically to 1 . In the inset we give $\sigma$ as a function of $d$ for different temperatures.

Fig. A shows how the fraction of frozen agents varies as the temperature is increased. For all values of $d$ there is a clear jump at $T=\mathcal{O}(1)$ from the MG value to $\phi=1$ [16]. Figs. 3 and 1 determine the phase diagram of the TMG in the $(d, T)$ plane. It is schematically depicted in the inset of Fig. 1. For low $d$ and $T$ the system performs worse than random, while for large enough values of $T$ 
the system becomes random, independently of $d$. Better than random performance is achieved between these two regions. It is important to note that, as in the case of the MG, the phase structure of the TMG depends strongly on the initial conditions. In particular, the low $(d, T)$ part of the phase diagram shrinks to zero for finite random initial conditions.

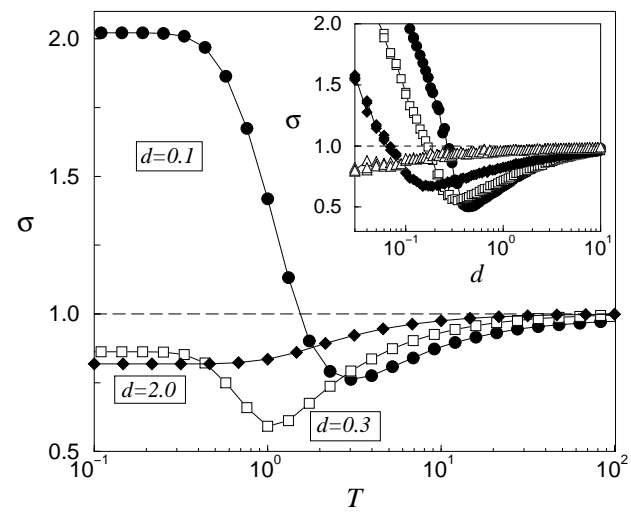

FIG. 3. Volatility as a function of the temperature from the continuous dynamics Eqs. (8). Inset: volatility as a function of $d$ for different values of the temperature $T=10^{-3}, 1,2,10(\bullet, \square, \diamond$, and $\triangle$, respectively). A second order stochastic Heun algorithm has been used with time step $d t=0.02$. Average over 20 realizations of the Wiener process and 50 samples; $N=100 ; t=t_{0}=10^{4}$; initial conditions $\mathbf{p}(t=0)=0$.

In the case where the probabilities are defined as $\pi_{i}^{1,2}(t) \propto \exp \beta P\left(\vec{R}_{i}^{1,2}, t\right)$, the monotonic increase of $\sigma$ to the random value at large $T$ reported in [13] is due to finite waiting times, as pointed out in [17]: for $t \gg N T$ the volatility stays at the minimum value for any finite $T \gg 1$. This phenomenon is easily understood from Eqs. (8). For large values of $T$, there is first a transient in which $s_{i}=\tanh \beta p_{i}$ are close to zero, and Eqs. (8) reduce to $d p_{i} \approx-h_{i} d t+\sum_{j k} J_{i k} J_{j k} d W_{j}$, i.e., the point differences of all the agents do a randomly biased Brownian motion, and the system performs as in the random case. Eventually, however, $p_{i}$ become of $\mathcal{O}(T)$ and $s_{i}$ finite, and the system effectively behaves as for $T \sim \mathcal{O}(1)$. Note that this cannot happen when $s_{i}=\tanh \beta z_{i}$.

Eqs. (8) are much simpler than the original ones for the microscopic description of the TMG. The external information has been replaced by an interaction among the agents and the random strategy choice has given rise to the diffusive term. They describe a dynamics which is different from the relaxation of disordered systems usually found in physical problems: the random force $\nabla_{\mathbf{s}} \mathcal{H}$ can be written as the gradient of a potential function only up to a factor, which amounts to a metric in the space of $\mathbf{p}$, and the non-trivial diffusion matrix $\mathbb{M}$ depends both on the variables $\mathbf{p}$ and on the quenched disorder of the problem. Finding adequate analytic asymptotic solutions to this dynamics is the next challenging task.
We thank J.-P. Bouchaud, A. Cavagna, I. Giardina, G. Lythe and D. Williams for useful discussions. This work was supported by EC Grant No. ARG/B7-3011/94/27 and EPSRC Grant No. GR/M04426.

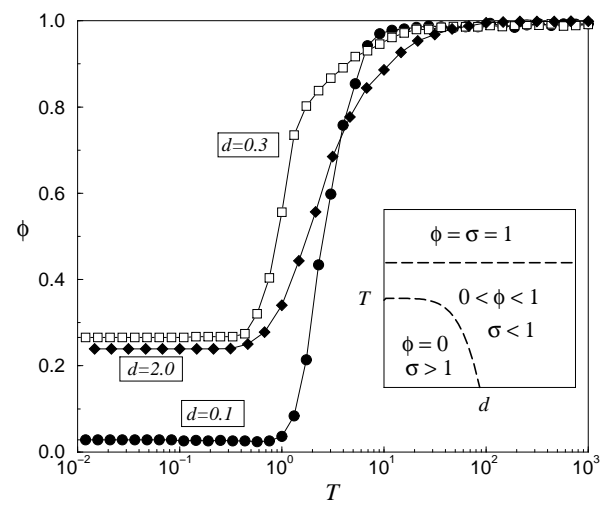

FIG. 4. Fraction of frozen agents $\phi$ as a function of $T$. Inset: schematic phase diagram of the TMG in the $(d, T)$ plane. Dashed lines indicate crossovers rather than sharp transitions.

a E-mail address: j.garrahan1@physics.ox.ac.uk.

$b \quad$ E-mail address: e.moro1@physics.ox.ac.uk.

c E-mail address: d.sherrington1@physics.ox.ac.uk.

[1] See, for example, Landscape Paradigms in Physics and Biology, edited by H. Frauenfelder et al., (North-Holland, Amsterdam, 1997).

[2] The Economy as an Evolving Complex System, edited by P.W. Anderson, K. Arrow and D. Pines (Addison-Wesley, Redwood City, 1988).

[3] J.D. Farmer, "Physicists attempt to scale the ivory towers of finance", e-print adap-org/9912002.

[4] D. Challet and Y.-C. Zhang, Physica A 246, 407 (1997).

[5] R. Savit, R. Manuca and R. Riolo, Phys. Rev. Lett. 82, 2203 (1999).

[6] W.B. Arthur, Science, 284, 107 (1999).

[7] N.F. Johnson, M. Hart and P.M. Hui, Physica A, 269, 1 (1999).

[8] R. D'hulst and G.J. Rodgers, Physica A, 270, 222 (1999).

[9] M. Hart, P. Jefferies, N. F. Johnson and P. M. Hui, "Crowd-anticrowd model of the Minority Game", condmat/0003486

[10] D. Challet and M. Marsili, Phys. Rev. E 60, R6271 (1999).

[11] D. Challet, M. Marsili and R. Zecchina, Phys. Rev. Lett. 84, 1824 (2000).

[12] A. Cavagna, Phys. Rev. E, 59, R3783 (1999).

[13] A. Cavagna, J.P. Garrahan, I. Giardina and D. Sherrington, Phys. Rev. Lett. 83, 4429 (1999).

[14] Any other normalized isotropic distribution in $\mathbb{R}^{D}$, e.g., a Gaussian, would be equally suitable. The same applies to the strategies.

[15] N.G. van Kampen Stochastic processes in physics and chemistry, (North-Holland, Amsterdam, 1992).

[16] When $T>0$, the fraction of agents playing always the same strategy is not given by $\phi$ but rather by $|\langle s\rangle| \propto$ $\phi \tanh \beta$.

[17] D. Challet, M. Marsili and R. Zecchina, Comment on 13, e-print cond-mat/0004308; A. Cavagna, J.P. Garrahan, I. Giardina and D. Sherrington, Reply, e-print condmat/0005134 